\title{
Controlling friction and wear with anisotropic microstructures in MoN coated surfaces
}

\author{
W. Schulz a), F. Köhn a), D. Kolb b), M. Balzer ${ }^{\text {c) }}$, H. Riegel b), J. Albrecht a) \\ a) Research Institute of Innovative Surfaces FINO, Aalen University, Beethovenstr. 1, D-73430 Aalen, Germany \\ b) LaserApplicationCenter LAZ, Aalen University, Beethovenstr. 1, D-73430 Aalen, Germany
}

c) Research Institute for precious Metals and Metal Chemistry fem, Department of Plasma Surface Technology and Materials Physics, Katharinenstr. 17, D-73525 Schwäbisch Gmünd

\begin{abstract}
Steel surfaces have been patterned by laser surface texturing (LST) to create anisotropic microstructures with typical dimensions of a few 10 micrometers. The subsequent deposition of thin molybdenum nitride coatings by high power impulse magnetron sputtering (HiPIMS) leads to surfaces that conserve the surface microstructures and exhibit an extraordinarily large resistance against mechanical wear. Tribological experiments with steel counter bodies show a substantial influence of the relative orientation of structures and wear track on friction and wear. It is pointed out that the main effect is the removal of abrasion particles from the mechanical contact. Analogue experiments with alumina counter bodies that hardly provide wear particles show that the orientation effect is absent.
\end{abstract}

\section{Introduction}

The protection of metallic surfaces against mechanical wear is frequently realized by the deposition of thin films of transition metal nitrides. In particular, titanium nitride (TiN) and titanium aluminum nitride (TiAIN) are typical materials used for industrial applications facing large mechanical forces. Recently, a growing interest on molybdenum nitride (MoN) is apparent because of its very high hardness and, in particular, its resistance against high temperatures above $250^{\circ} \mathrm{C}$ [1-3]. Since the formation of stoichiometric phases in the molybdenum-nitrogen system require a deposition process far from thermal equilibrium typically high power impulse magnetron sputtering (HiPIMS) [4], arc plasma-assisted deposition [5] or pulsed laser deposition (PLD) [6] are used for the coating process.

A further increase the extraordinary wear resistance of hard surfaces can be obtained by additional surface treatments such as the introduction of microstructures into the surface. In particular, this is found to be useful in case of dry contacts [7]. There are various methods of producing suitable surfaces for reducing friction. Shimizu et al. structured brass surfaces with vibrationassisted microcutting [8]. Various micro/nanoscale geometries can be produced by a single crystal diamond tool through elliptical vibration cutting [9]. Sube et al. have created microimprints on the surface by mechanical embossing using a diamond stamp, significantly reducing the occurring friction against steel [10]. It has been shown that a modification of the surface topography on a length scale of several ten micrometers prepared by laser structuring or mechanical imprinting can efficiently reduce friction and wear [11-19]. In case of an abrasion dominated wear mechanism the main role is to capture wear debris inside the structures to remove it from the mechanical contact. Kumar et al. showed that by microstructuring the surface abrasion particles are trapped and wear is reduced [20]. Furthermore, Kommer et. al found that the combination of prior structuring of the surface with subsequent deposition of a hard material layer additionally reduces wear. In addition, accumulating material inside the microimprints during wear tests has been identified to be originated by the steel counter body [4]

In this work we consider anisotropic, line-like surface structures that created by laser structuring in steel surfaces before coating with molybdenum nitride. Similar surface modifications have been considered by Rosenkranz et al. for the investigation of steel/steel contacts [11]. It has been found that wear debris is substantial for the description of friction and wear. Distribution and kinetics of wear debris is strongly affected by the relative orientation of structures and wear track. In addition, a systematic analysis of the orientation dependence of friction and wear of the surface structures is performed. This ansatz is transferred to nitride coated surfaces in this work. The results of Rosenkranz et al. give rise to the idea that capturing and guiding of abrasion particles provided by the counter body can significantly control occurring friction and wear at MoN surfaces against steel.

\section{Experimental details}

We use small blocks made of high-speed steel (DIN 1.3343) with dimensions of $20 \times 12 \times 3 \mathrm{~mm}^{3}$. The surface has been ground and polished using standard preparation methods to achieve a final roughness of $R_{a}=5 \mathrm{~nm}$. The line structures have been produced using an infrared TruMark 5020 marking laser from TRUMPF. A width of approx. $45 \mu \mathrm{m}$ was selected as focal diameter and the line spacing has been chosen to be $100 \mu \mathrm{m}$. The feed rate of the laser is $800 \mathrm{~mm} / \mathrm{s}$ at an 
average laser power of $20 \mathrm{~W}$ and a pulse frequency of $80 \mathrm{kHz}$. The crossing is carried out twice at each surface to ensure an appropriate aspect ratio of the lines. Subsequently, the samples were again subjected to a gentle polishing process to remove the accumulated material along the line edges to obtain sharp edges. After polishing the specimens were cleaned ultrasonically in an acetone bath for $10 \mathrm{~min}$.

The MoN coatings were deposited by reactive high power impulse magnetron sputtering (HiPIMS) using a 3" sputtering cathode with a Mo-target (purity 99.95 at. $-\%)$. The process parameters were chosen to realize a pure $\delta$-MoN coating as first published by Kommer et al. [5]: HiPIMS-Pulse pattern: $t_{\text {on }}=14 \mu \mathrm{s}$, $\mathrm{T}=2000 \mu \mathrm{s}, \quad \mathrm{p}\left(\mathrm{N}_{2}\right)=0.4 \mathrm{~Pa}, \quad \mathrm{p}\left(\mathrm{Ar}+\mathrm{N}_{2}\right)=0.6 \mathrm{~Pa}$. No external substrate bias voltage has been applied. The coating thickness was about $1.8 \mu \mathrm{m}$ as measured by tactile profilometry on a coating step gained by covering a part of the substrate with a razor blade segment. The indentation hardness was $H_{I T}$ $(0.009 / 10 / 10 / 10)=32 \pm 2$ GPa (Fischer H100 xy p).

The topography of the surfaces before and after deposition of the nitride layers are mapped by whitelight interferometry and light microscopy. Results are depicted in Figure 1.

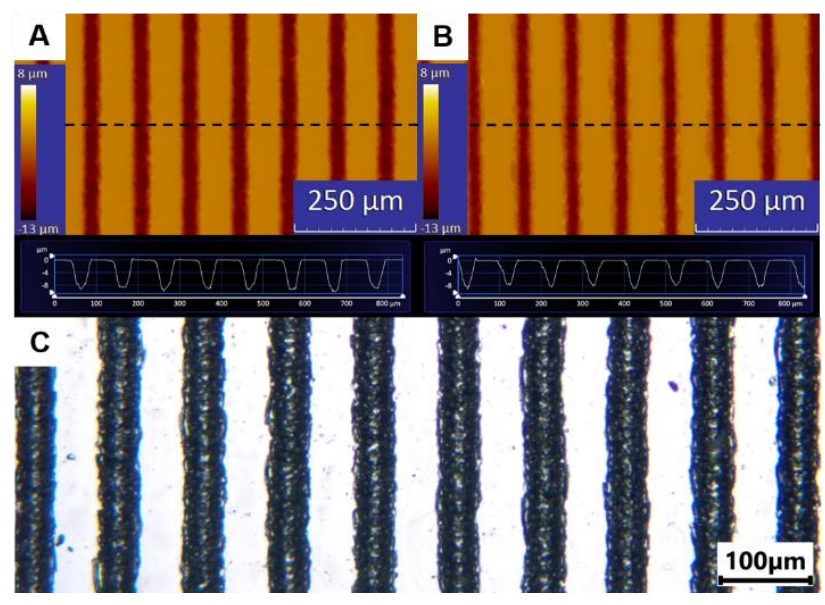

Figure 1: Surface topography of line-like surface structure obtained by Laser patterning. The results are obtained by white-light interferometry before $(A)$ and after $(B)$ the deposition of MoN. The profiles below are extracted along the horizontal dashed lines (C) Optical micrograph of a the surface after coating.

Panels (A) and (B) show images of a linearly structured surface before and after deposition, respectively. In addition, a corresponding horizontal profile is depicted below. We find that the PVD process basically conserves the prepared laser structure after deposition. Both images show a highly regular structure of parallel lines with an individual width of about $40-45 \mu \mathrm{m}$ and a depth of $8-9 \mu \mathrm{m}$. However, a close look reveals that both width and depth of the created linear structures are slightly reduced by about $10 \%$ after deposition. Since the depth of the laser structures is significantly larger than the film thickness of the MoN layer this does not affect further conclusions of this work.

Additional information is gained from the optical micrograph (C) that is depicted below. Here, it is seen that the lines of removed material exhibit an irregular microstructure that is basically originated by the Laser preparation process in pulse mode. The surface areas in between the structured regions remain basically unmodified indicating that the parts of the surface that will be in mechanical contact with a counter body are hardly modified.

To access the required tribological information, namely friction and wear, all structured and coated surfaces were thoroughly characterized by an oscillation tribometer from Optimol SRV3. The set-up is equipped with $100 \mathrm{Cr} 6$ (Hardness $6.9 \mathrm{GPa}$ ) and $\mathrm{Al}_{2} \mathrm{O}_{3}$ (Hardness $16.7 \mathrm{GPa}$ ) balls as counter bodies with a diameter of $10 \mathrm{~mm}$ that are pressed onto the surface using a load of $\mathrm{F}_{\mathrm{N}}=20 \mathrm{~N}$. During all measurements a stroke length of $1 \mathrm{~mm}$, a frequency of $2 \mathrm{~Hz}$ and a temperature $\mathrm{T}=50^{\circ} \mathrm{C}$ are used. The exposure duration has been $t=900 \mathrm{~s}$ in each case. After mechanical treatment, shape, width and volume of the wear track are analyzed using white-light interferometry. Every experiment has been repeated three times.

\section{Results}

First, the coefficients of friction are measured against steel counter bodies under dry conditions. In particular, the relative orientation of surface pattern and wear track is varied systematically. The results are depicted in Figure 2.

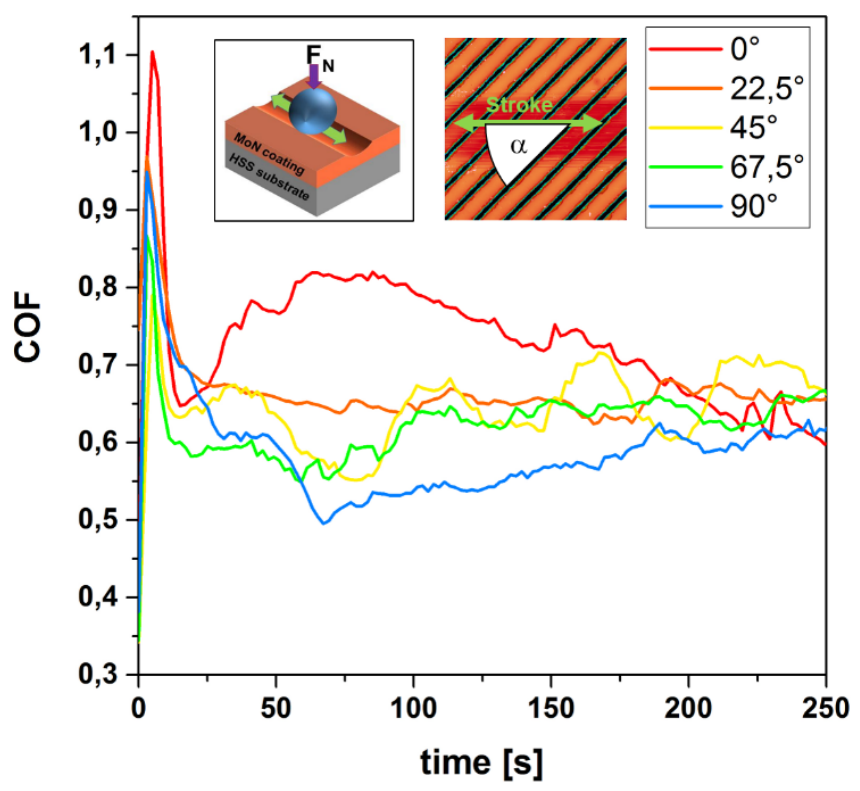

Figure 2: Average coefficients of friction (COF) with a 100Cr6 counter body against a structured MoN coating up to $250 \mathrm{~s}$. Inset left: schematic representation of the tribological stress. Inset center: determination of the angles in relation to the loading direction. 
The figure shows the friction coefficients obtained under relative angles of $0^{\circ}$ to $90^{\circ}$ in steps of $22.5^{\circ}$. Each curve refers to an average of three individual measurements. Inset 1 (top left) schematically shows the mechanical stress during the tribometry testing. Here, a spherical counter body is guided over the surface in oscillating motion under exclusion of a lubricant. The definition of the angle $\alpha$ is found in Inset 2 (center). $\alpha=0^{\circ}$ denotes a wear track parallel to the line-like structures, for $\alpha=90^{\circ}$ the structures are perpendicular to the motion of the counter body.

The results of the friction experiments are shown as colored curves over a time period of $t=250 \mathrm{~s}$. Different colors refer to different angles. After a short initial period of about $t=25 \mathrm{~s}$ that is not considered any further we find a systematic decrease of the COF with increasing angle $\alpha$. The strongest effect is found around $\mathrm{t}=75 \mathrm{~s}$ where a large COF of $\mu=0.82$ is found for an angle of $\alpha=0^{\circ}$ that continuously decreases to $\mu=0.49$ in case of the $90^{\circ}$ geometry. After $t=75 \mathrm{~s}$ the difference of the individual curves gradually decreases up to $t=225 \mathrm{~s}$. After that point of time no significant difference of the individual COF curves can be detected.

In order to determine the wear volume of layer and counter body the wear tracks have been characterized using white light interferometry. An overview of the results is depicted in Figure 3.

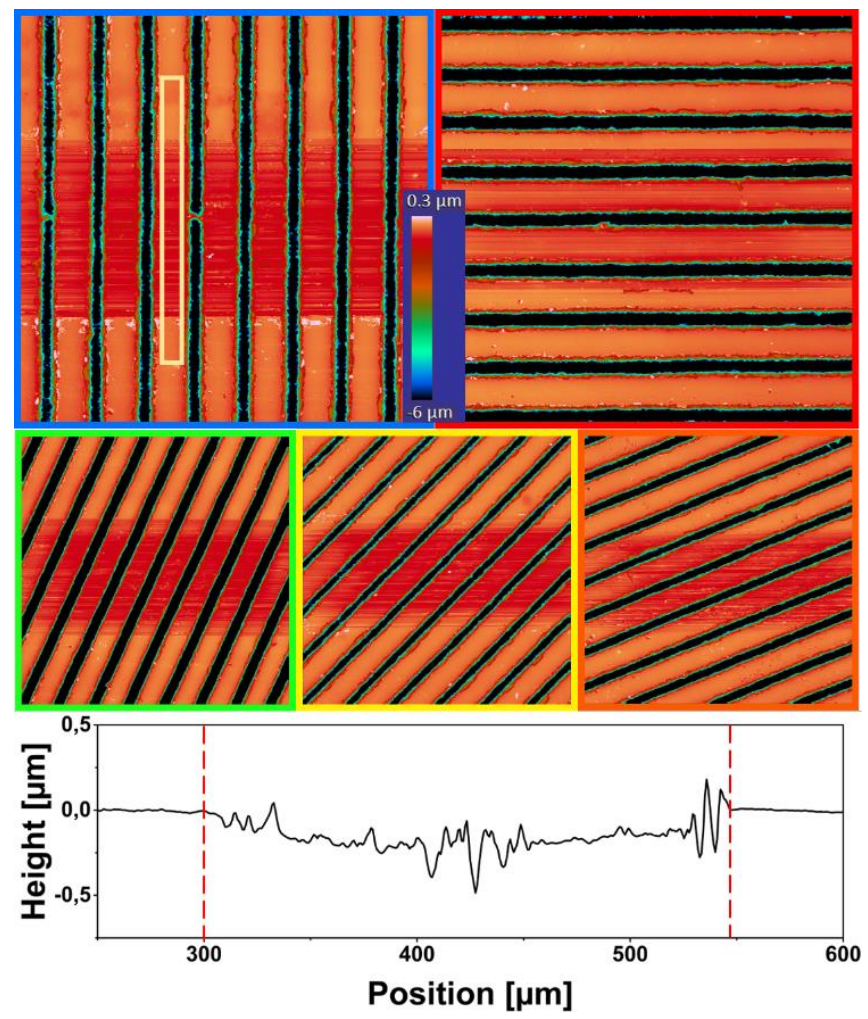

Figure 3: Profilometric images after tribological characterization. The top row depicts parallel and perpendicular orientation: $90^{\circ}$ (left, blue); $0^{\circ}$ (right, red) The second row depict the angles in between: green box: $67.5^{\circ}$ (left, green); $45^{\circ}$ (center, yellow); $22.5^{\circ}$ (right, orange). The profile below is extracted from the marked box in the top left image. Vertically oriented, dashed lines mark the width of the wear track.

Figure 3 shows interferometry images of the wear tracks occurring in the individual structured surfaces $\left(\alpha=0^{\circ}-90^{\circ}\right)$ after tribological characterization over $\mathrm{t}=900 \mathrm{~s}$. The introduced Laser structures are clearly visible as dark parallel lines. The top row depicts the cases of parallel and perpendicular orientation: $\alpha=90^{\circ}$ (top left) and $\alpha=0^{\circ}$ (top right). Below, the wear tracks of the angles $\alpha=67.5^{\circ}$ (bottom left), $\alpha=45^{\circ}$ (bottom center) and $\alpha=22.5^{\circ}$ (bottom right) are shown. The depth information is shown by the color scaling in the color bar at the center, with a spectrum from 0.3 (white) to $-6 \mu \mathrm{m}$ (black). All images allow the identification of the wear track that is seen as red stripe with horizontal orientation. In all cases a width of about $250 \mu \mathrm{m}$ is found, however, it continuously decreases slightly from $90^{\circ}$ to $0^{\circ}$ by roughly $10 \%$.

The depth information can also be extracted from the interferometry images. As an example, a profile is depicted below that is extracted from the yellow box in the top left panel of Figure 3. Here, a wear track with basically spherical shape is found exhibiting a width of $250 \mu \mathrm{m}$. In addition, the profile is not smooth. There are individual narrow wear scars with a typical width of about $10 \mu \mathrm{m}$ and a depth of several $100 \mathrm{~nm}$. We find a maximum depth of the wear profile of about $0.5 \mu \mathrm{m}$ clearly indicating that the wear is restricted to the MoN layer with a thickness of $1.8 \mu \mathrm{m}$.

To determine the wear volume in the MoN film the analysis uses an averaged profile perpendicular to the wear track. Then the surface fraction has to be considered that has been removed during Laser structuring and where no material has been removed.

An exceptional procedure has to be performed for the $0^{\circ}$ geometry. Here, an averaged profile does not provide the correct shape of the wear track. The missing volume has to be extracted individually from each area between the Laser lines as depicted in Figure 4.

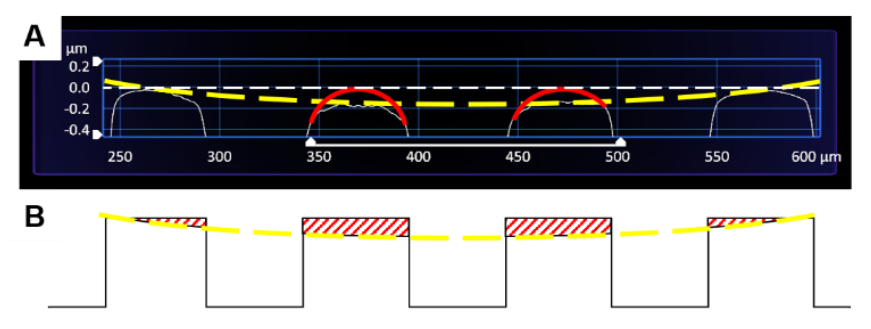

Figure 4: Original wear profile at $\alpha=0^{\circ}$ as measured (A). The white dashed line marks the surface before the testing. The yellow dashed line shows the spherical wear track, the red ball caps indicate the areas where material is removed. Schematic representation of the profile for better determination of the wear volume (B). 
An exception is the structure at $\alpha=0^{\circ}$. Owing to the parallel direction of motion material removal occurs only at some individual ridges between the Laser lines as shown in Figure $4(A)$. Here, the missing volume has to be determined locally at these ridges. Therefore, a rectangular surface topography has been assumed leading to some enlarged error bars in the final evaluation. The performed process is shown schematically in Figure 4 (B).

Wear volume occurring at the ball-shaped counter body has been extracted geometrically. The diameter of the flat and circular-shaped wear region has been extracted. The wear volume is then calculated as the volume of the missing ball cap. In case of the $0^{\circ}$ result, the wear volume cannot be described by a spherical cap due to the reasons above. Again, a local investigation has to be performed ending in values with larger error bars. A sum of the determined wear volumes is depicted in Figure 5. For comparison, values for the COF are added that are extracted from Figure 2 by averaging between $\mathrm{t}=25 \mathrm{~s}$ and $\mathrm{t}=225 \mathrm{~s}$.

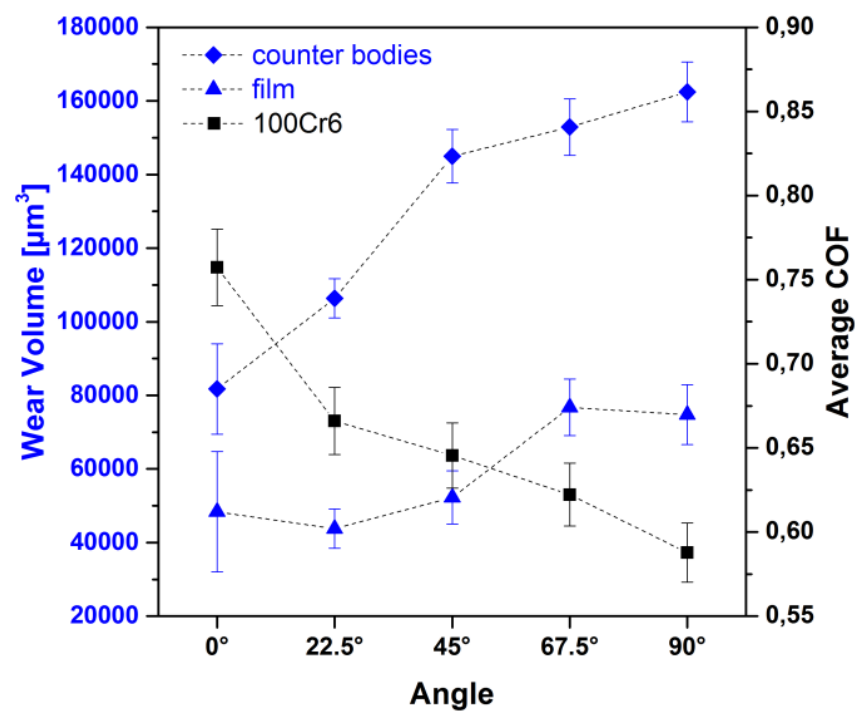

Figure 5: Angular dependence of the wear volumes of the MoN layer and the counter body (blue). Average coefficients of friction (black).

By changing the orientation of the structures to higher angles the wear volume also increases. In the case of the MoN film (blue triangles) a wear volume of $40,000 \mu \mathrm{m}^{3}$ to $75,000 \mu \mathrm{m}^{3}$ is found. A similar behavior shows the wear volume of the counter body (blue diamonds). Here the values are between $80,000 \mu^{3}$ and $160,000 \mu^{3}$. The wear of the counter bodies is approx. $100 \%$ higher compared to the microstructured hard coating. The black squares describe the average coefficient of friction during $t=25 \mathrm{~s}$ and $t=225 \mathrm{~s}$ extracted from the friction curves shown in Figure 2. It was observed that with increasing angle of the structures the coefficient of friction decreases.

Owing to the large wear of the counter body it is assumed that the observed damage is significantly caused by third particles originated by the $100 \mathrm{Cr} 6$ steel counter body. These particles are notably responsible for friction and wear. The observed systematic influence of line-like surface structures is closely related to the behavior of these particles inside the tribological contact.

To verify this assumption, we perform an experiment using an $\mathrm{Al}_{2} \mathrm{O}_{3}$ counter body that exhibits a significantly higher wear resistance and, thus, provides hardly any abrasion particles. Using the same parameters as for the results in Figure 2 we obtain friction curves that are depicted in Figure 6.

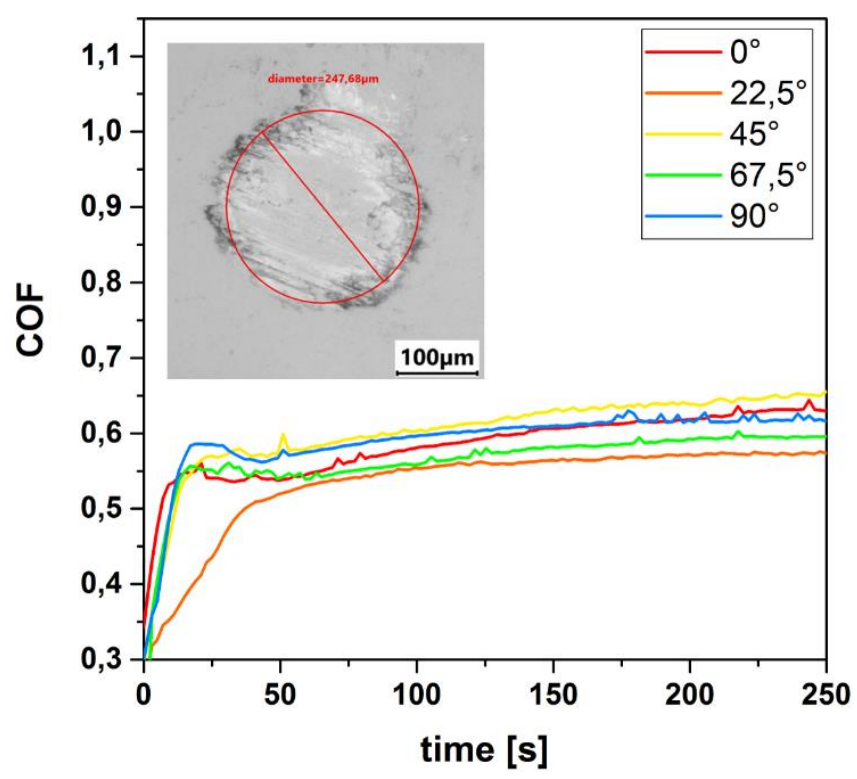

Figure 6: Coefficients of friction with $\mathrm{Al}_{2} \mathrm{O}_{3}$ counter body against a structured MoN coating up to $250 \mathrm{~s}$ under equivalent conditions as used for Figure 2. Inset: Optical micrograph of an $\mathrm{Al}_{2} \mathrm{O}_{3}$ counter body after the tribological test.

The measurements in Figure 6 are shown with the same color code as in Figure 2. Here, a significant difference to the results of Figure 2 is obvious. All obtained friction curves show basically the same behavior. After the initial period of $t=25 \mathrm{~s}$ all curves start at coefficient of friction values of $\mu=0.50-0.55$. During the measurement period of $250 \mathrm{~s}$ a slight increase up to values of $\mu=0.55-0.60$ is observed. No systematic dependence can be found when varying the orientation of the microstructures. This is in clear contrast to the experiments using a $100 \mathrm{Cr} 6$ counter body.

For a more detailed analysis of the found friction results we summarize the obtained average coefficients of friction for $100 \mathrm{Cr} 6$ and $\mathrm{Al}_{2} \mathrm{O}_{3}$ counter bodies in Figure 7 . 


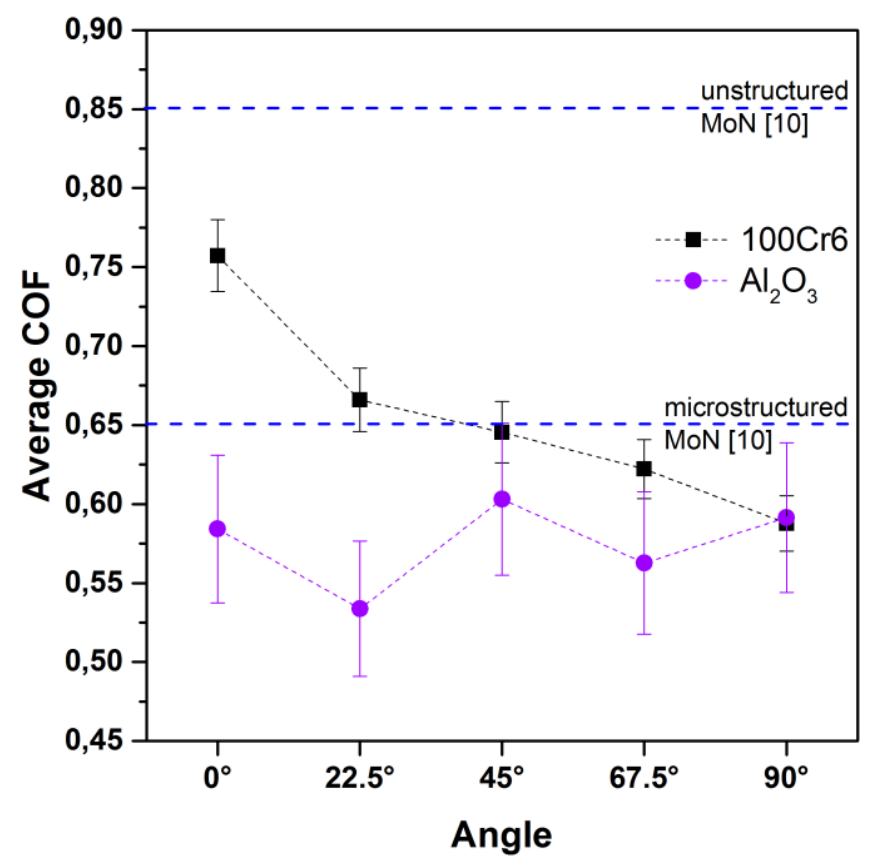

Figure 7: Coefficients of friction of $100 \mathrm{Cr} 6$ (black) and Al2O3 (purple) counter bodies against MoN as a function of the angle of the microstructures. For reference: unstructured MoN coated surface (upper dashed line) and microimprinted MoN coated surface (lower dashed line) versus $100 \mathrm{Cr} 6$ counter bodies [10].

The black curve refers to the $100 \mathrm{Cr} 6$ counter bodies, the purple curve to $\mathrm{Al}_{2} \mathrm{O}_{3}$. As already described a decrease of the friction values with increasing angle can be observed against the steel counter body. The friction in case of the motion parallel to the Laser lines exhibits a maximum at $\mu=0.76$, the smallest value wit $\mu=0.59$ is found for the perpendicular orientation. Changing the set-up to an $\mathrm{Al}_{2} \mathrm{O}_{3}$ counter body, the friction values range from $\mu=0.53-0.60$ and no systematic dependence can be extracted. In addition, the coefficient of friction values for unstructured and microimprinted MoN coated surfaces versus $100 \mathrm{Cr} 6$ are added to the diagram as dashed lines. The upper dashed line describes friction values of a similar surface without structure. Average friction values of approx. $\mu=0.85$ are achieved here. A similar experiment was carried out by Sube et al. using a stamp to structuring the sample surface. A MoN layer was then deposited and the surface tribologically characterized [10]. The average coefficient of friction against a $100 \mathrm{Cr} 6$ counter body was also approx. $\mu=0.65$, shown as the lower dashed line in Figure 7.

The different behavior of the friction curves for both counter bodies shows that the systematic influence of orientation is related to the occurrence of abrasion particles. Determining the abrasion volume after mechanical testing at the counter bodies by interferometry it is found that the steel counter bodies exhibit an average wear volume of the order of $100,000 \mathrm{\mu m}^{3}$, in case of alumina values of approx. $2,000 \mu \mathrm{m}^{3}$ are found. This means that the number of abrasion particles is drastically reduced in the $\mathrm{Al}_{2} \mathrm{O}_{3}$ contact.

\section{Discussion}

It is known, that suitable surface structures are able to remove third particles out of the frictional contact and decrease friction and wear. Kumar et al. created structures on uncoated $100 \mathrm{Cr} 6$ steel surfaces by laser and characterized them with respect to friction and wear. With such structures it is possible to remove third bodies from the tribological system by trapping them inside the cavities. The structure alone led to a reduction in the coefficient of friction from approx. $\mu=0.70$ to approx. $\mu=0.55$ [20]. Sube et al. showed that it is possible to significantly reduce friction by combining surface structuring and a hard material layer. Here, a reduction of the coefficient of friction by $25 \%$ could be achieved (see blue dashed lines in Figure 7) [10].

The presented results confirm the impact of structured surfaces on friction and wear. The significance of the results is large since completely identical surfaces have been examined, differing only in the orientation of the introduced structures. The particles generated are trapped in the line-like structures and do not contribute in the frictional contact. The orientation of the lines is to emphasize here. In case of parallel orientation $\left(\alpha=0^{\circ}\right)$ the highest coefficient of friction was observed, as shown in Figure 2. A stepwise reorientation of the structure ensured a reduction of the observed COF down to a value of $\mu=0.60$. Comparing the line structures with the structured surface of Sube et al. it could be shown that equivalent coefficients of friction are already obtained from an orientation of $\alpha=45^{\circ}$, Figure 7. With a perpendicular orientation $\left(\alpha=90^{\circ}\right)$ the effect could be increased by further $10 \%$.

As already observed by Kumar et al. the effect of particle trapping is limited in time. Due to the relatively large surface stress and the absence of a lubricant in the tribological contact the structures are repeatedly clogged or, in the worst case, completely abraded [20]. The same behavior could be observed in these experiments. Although the respective tribological tests had a total duration of $900 \mathrm{~s}$, the friction values from Figure 2 reached a similar plateau after only about $225 \mathrm{~s}$. This suggests that from this point on, the structures were clogged with particles and the positive effect was no longer effective. Topographic investigations confirmed this assumption. It was observed that inside the wear track the line structures were clogged with particles. All data points after $\mathrm{t}=225 \mathrm{~s}$ have been omitted for the analysis.

Since the wear volume is directly related to the presence of abrasive particles the wear on the 
respective surfaces has been examined in more detail. The lowest volumes are achieved with a structure orientation below $\alpha=45^{\circ}$.

The steel counter body moves along the structure in the case of $\alpha=0^{\circ}$ orientation, similar to rails. The effective area for generating abrasion particles is smaller than for the other surface structures. In addition, the abrasion of the counter body leads to a grooved surface where the remaining parts fit into the lines of the MoN film. This increases the mechanical contact area and the role of particles. Thus, the coefficient of friction increases.

Within the structure, the particles are transported out of the wear track by the relatively high shear forces until they become anchored in the structure by mechanical interlocking and level the structure.

The situation is similar at $\alpha=45^{\circ}$. Here, the wear volume is at a similar level as for smaller angles. Significantly higher wear volumes were observed for orientations above $\alpha=45^{\circ}$. Up to $100 \%$ more coating material was removed than at small angles.

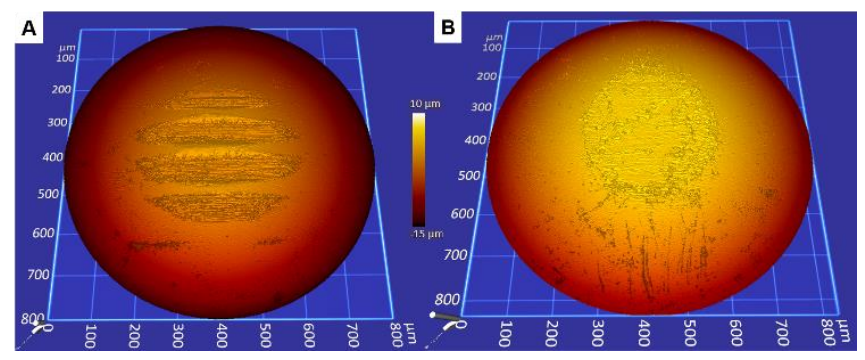

Figure 8: White light interferometric images of 100Cr6 counter bodies after tribological loading. A: Wear with the structures oriented parallel $\left(\alpha=0^{\circ}\right)$ to the direction of motion. B: Wear with structure orientations $\alpha>0^{\circ}$.

The same investigations were carried out on the steel counter bodies. Here, the direct influence of the structure in the abrasion volume can be seen. With a parallel structure alignment $\left(\alpha=0^{\circ}\right)$ in relation to the direction of motion, rather small abrasion takes place on the counter bodies. This is justified in the comparatively small contact area of the friction partners leading to a striking wear pattern on the counter bodies, as shown in Figure 8 (A). As an example, the exemplary removal at all other angles is shown in Figure 8 (B). It is seen that a flat surface has evolved at the end of the testing period. Changing the orientation to larger angles the erosion increases up to a value of $160,000 \mu^{3}$ which corresponds to an increase in wear of about $100 \%$. The relatively sharp edges of the structures lead to an effect similar to the machining of metals. Individual layers are scraped off the counter bodies during each cycle resulting in the damage patterns shown. This effect increases with increasing angle of the surface structures.

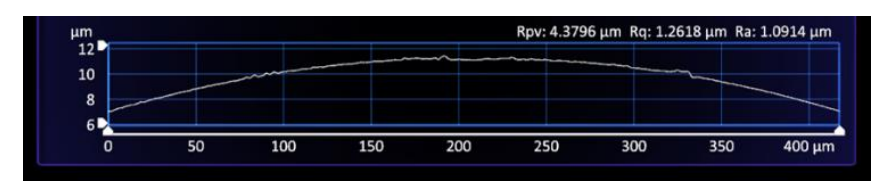

Figure 9: Surface profile of an $\mathrm{Al}_{2} \mathrm{O}_{3}$ counter body after tribological testing.

For comparison, counter bodies made of $\mathrm{Al}_{2} \mathrm{O}_{3}$ were used to confirm the influence of particle trapping. The friction curves depicted in Figure 6 show an almost identical course from a duration of about $30 \mathrm{~s}$ onward. A comparison of the ceramic and steel counter bodies shows that the friction values of $\mathrm{Al}_{2} \mathrm{O}_{3}$ range from $\mu=0.53-0.60$ and are thus generally lower. Here, the influence of the structure seems to be only marginal. The surface of the $\mathrm{Al}_{2} \mathrm{O}_{3}$ counter bodies was characterized after testing using a white light interferometer. It was found that hardly any abrasion took place on the counter bodies, as can be seen in Figure 9.

\section{Conclusions}

Wear resistive surfaces with anisotropic line-like microstructures are produced in a two-step process. First, Laser patterning is used to create regular microgrooves into a steel surface. In a second step the steel surfaces are coated with MoN by HiPIMS. It is shown that the structures introduced are an effective tool to control friction and wear at these surfaces. A change of the relative angle of structures and motion leads to a systematic change of friction and wear. In particular, effective removing of friction particles from the tribological contact and thus reducing coefficient of friction is possible. This is most successful if the line structures are oriented perpendicular to the load direction. A comparison with microembossed surfaces from literature [10] shows that the structures used here, with suitable orientation, can enable a previously unattained reduction in the coefficient of friction. A comparison with $\mathrm{Al}_{2} \mathrm{O}_{3}$ counter bodies shows that the underlying mechanism is the trapping of the abrasion particles of the counter bodies.

\section{Acknowledgment}

The authors would like to thank Gaby Ketzer-Raichle and Elvira Reiter of the Institute for Materials Research Aalen (IMFAA) for their active support in the preparation of the specimens.

\section{References}

[1] G. Gassner, P.H. Mayrhofer, K. Kutschej, C. Mitterer, M. Kathrein, Magnéli phase formation of PVD Mo-N 
and W-N coatings, Surface and Coatings Technology 201 (6) (2006) 3335-3341.

[2] T. Suszko, W. Gulbiński, J. Jagielski, The role of surface oxidation in friction processes on molybdenum nitride thin films, Surface and Coatings Technology 194 (2-3) (2005) 319-324.

[3] R.A. Koshy, M.E. Graham, L.D. Marks, Synthesis and characterization of $\mathrm{CrN} / \mathrm{Mo} 2 \mathrm{~N}$ multilayers and phases of Molybdenum nitride, Surface and Coatings Technology 202 (4-7) (2007) 1123-1128.

[4] M. Kommer, T. Sube, A. Richter, M. Fenker, W. Schulz, B. Hader, J. Albrecht, Enhanced wear resistance of molybdenum nitride coatings deposited by high power impulse magnetron sputtering by using micropatterned surfaces, Surface and Coatings Technology 333 (2018) 1-12

[5] O.V. Krysina, Yu.F. Ivanov, N.N. Koval, N.A. Prokopenko, V.V. Shugurov, E.A. Petrikova, O.S. Tolkachev, Composition, structure and properties of Mo-N coatings formed by the method of vacuum-arc plasma-assisted deposition, Surface and Coatings Technology 416 (2021) 127153.

[6] M. Bereznai, Z. Tóth, A.P. Caricato, M. Fernández, A. Luches, G. Majni, P. Mengucci, M. Nagy, A. Juhász, L. Nánai, Reactive pulsed laser deposition of thin molybdenum- and tungsten-nitride films, Thin Solid Films, 473 (2005) 16-23.

[7] C. Gachot, A. Rosenkranz, L. Reinert, E. RamosMoore, N. Souza, M. H. Müser, F. Mücklich, Dry Friction Between Laser-Patterned Surfaces: Role of Alignment, Structural Wavelength and Surface Chemistry, Tribology Letters 49 (2013) 193-202

[8] J. Shimizu, T. Nakayama, K. Watanabe, T. Yamamoto, T. Onuki, H. Ojima, L. Zhou, Friction characteristics of mechanically microtextured metal surface in dry Sliding, Tribology International, 149 (2020) 105634.

[9] J. Zhang, J. Zhang, A. Rosenkranz, N. Suzuki, E. Shamoto, Frictional properties of surface textures fabricated on hardened steel by elliptical vibration diamond cutting, Precision Engineering 59 (2019) 6672.

[10] T. Sube, M. Kommer, M. Fenker, B. Hader, J. Albrecht, Reduced friction ony-Mo2N coatings deposited by high power impulse magnetron sputtering on microstructured surfaces, Tribology International 106 (2017) 41-45.

[11] A. Rosenkranz, L. Reinert, C. Gachot, F. Mücklich, Alignment and wear debris effects between laser- patterned steel surfaces under dry sliding conditions, Wear 318 (1-2) (2014) 49-61.

[12] J. Oksanen, T. J.Hakala, S. Tervakangas, P. Laakso, L. Kilpi, H. Ronkainen, J. Koskinen, Tribological properties of laser-textured and ta-C coated surfaces, Tribology International 70 (2014) 94-103.

[13] A. Amanov, R. Tsuboi, H. Oe, S. Sasaki, The influence of bulges produced by laser surface texturing on the sliding friction and wear behavior, Tribology Interntional 60 (2013) 216-223.

[14] P. Andersson, J. Koskinen, S. Varjus, Y. Gerbig, H. Haefke, S. Georgiou, B. Zhmud, W.Buss, Microlubrication effect by laser-textured steel surfaces, Wear 262 (3-4) (2007) 369-379.

[15] R. Bathe, V. Sai Krishna, S. K. Nikumb, G. Padmanabham, Laser surface texturing of gray cast iron for improving tribological behavior, Applied Physics A 117 (1) (2014) 117-123.

[16] A. Higuera-Garrido, R. Gonza'lez, M. Cadenas, J. L. Viesca, A. Herna'ndez-Battez, Microstructure of $\mathrm{NiCrBSi}$ laser cladding coatings textured by ND-YAG laser, Proceeding of the ASME/STLE 2011 International Joint Tribology Conference (2011) 61088.

[17] G. Fiaschi, M. Di Lauro, A. Ballestrazzi, A. Rota, F. Biscarini, S. Valeri, Tribological response of lasertextured steel pins with low-dimensional micrometric patterns, Tribology International 149 (2020) 105548.

[18] C. Gachot, P. Grützmacher, A. Rosenkranz, Laser Surface Texturing of TiAl Multilayer Films-Effects of Microstructure and Topography on Friction and Wear, Lubricants 6 (2) (2018) 36.

[19] T. Stark, S. Alamri, A. I. Aguilar-Morales, T. Kiedrowski, A. F. Lasagni, Positive Effect of Laser Structured Surfaces on Tribological Performance, JLMN-Journal of Laser Micro/Nanoengineering Vol. 14 (1) (2019) 13-18.

[20] M. Kumar, V. Ranjan, R. Tyagi, Effect of Shape, Density, and an Array of Dimples on the Friction and Wear Performance of Laser Textured Bearing Steel under Dry Sliding, Journal of Materials Engineering and Performance, 29 (5) (2020) 2827-2838. 